\title{
Valutazione economica dello studio PROVE-IT
}

Lorenzo G. Mantovani ${ }^{(1)}$, Sabato Montella ${ }^{(1)}$, Anna Citarella $^{(1)}$, Simona de Portu ${ }^{(1)}$

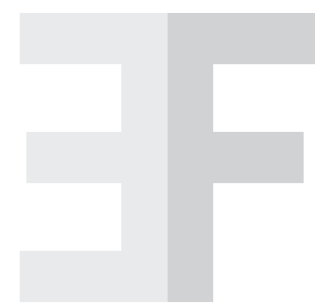

\begin{abstract}
Introduction: the PROVE-IT ("Intensive versus moderate lipid lowering with statins after acute coronary syndromes") was a comparison of pravastatin $40 \mathrm{mg} /$ die versus atorvastatin $80 \mathrm{mg} /$ die in patients with an acute coronary syndrome (ACS).

Aim: our aim was to investigate the economic consequence of high dose of atorvastatin vs usual-dose of pravastatin in Italian patients with a history of acute coronary syndrome.

Methods: the analysis is conducted on the basis of clinical outcomes of the PROVE-IT study.

We conducted a cost-effectiveness analysis, comparing high dose of atorvastatin $(80 \mathrm{mg} / \mathrm{die})$ versus usual-dose of pravastatin $(40 \mathrm{mg} / \mathrm{die})$ in the perspective of the Italian National Health Service.

We identified and quantified medical costs: drug costs according to the Italian National Therapeutic Formulary and hospitalizations were quantified based on the Italian National Health Service tariffs (2006). Effects were measured in terms of mortality and morbidity reduction (number of deaths, life years gained and frequency of hospitalizations).

We considered an observation period of 24 months.

The costs borne after the first 12 months were discounted using an annual rate of $3 \%$. We conducted one and multi-way sensitivity analyses on unit cost and effectiveness. We also conducted a threshold analysis.

Results: the cost of pravastatin or atorvastatin therapy over the 2 years period amounted to approximately 1.3 millions euro and 870,000 euro per 1,000 patients respectively. Atorvastatin was more efficacious compared to pravastatin and the overall cost of care per 1,000 patients over 24 months of follow-up was estimated at 3.2 millions euro in the pravastatin and 2.5 millions euro in the atorvastatin group, resulting into a cost saving of about 700,000 euro that is $27 \%$ of total costs occurred in the pravastatin group.

Discussion: this study demonstrates that high does atorvastatin treatment leads to a reduction of direct costs for the National Health System. Atorvastatin therapy is dominant since it is both less costly and more effective than pravastatin. Results of sensitivity analysis showed that atorvastatin therapy remains dominant even in the most unfavourable hypotheses.
\end{abstract}

Keywords: atorvastatin, pravastatin, acute coronary syndrome, cost, Italian National Health System

\section{INTRODUZIONE}

Gli effetti sulla riduzione del rischio di eventi cardiovascolari in pazienti con sindrome coronarica acuta sono stati recentemente valutati dallo studio Intensive versus moderate lipid lowering with statins after acute coronary syndromes (PROVE-IT) che ha confrontato due strategie terapeutiche atte a ridurre il livello di colesterolo: pravastatina a dosaggi standard versus atorvastatina a dosaggi elevati [1].

Lo studio ha dimostrato come, nei pazienti con sindrome coronarica acuta, un regime terapeutico ipolipemizzante maggiormente aggressivo riduce il rischio successivi eventi coronarici maggiori più di quanto non faccia un regime terapeutico a dosaggi standard.

Sulla base di tali considerazioni il nostro studio si propone di valutare l'impatto economico in Italia dell'utilizzo di dosi elevate di atorvastatina versus pravastatina a dosi standard in pazienti con coronaropatia.

\section{METODI}

Le informazioni cliniche sono state tratte dallo studio PROVE-IT che ha valutato gli effetti di due differenti strategie terapeutiche (atorvastatina $80 \mathrm{mg} /$ die vs pravastatina 40 $\mathrm{mg} / \mathrm{die}$ ) sul rischio di morte per cause cardiovascolari maggiori [1].

In tale studio sono stati arruolati 4.162 pazienti di età superiore ai 18 anni, ospedalizzati nei 10 giorni precedenti l'arruolamento per sindrome coronarica acuta, infarto del miocardio o angina instabile. I pazienti che rispettavano i criteri di eleggibilità sono stati randomizzati a ricevere pravastatina $40 \mathrm{mg} / \mathrm{die}(2.063) \mathrm{o}$ atorvastatina $80 \mathrm{mg} / \mathrm{die}(2.099)$.
${ }^{(I)}$ CIRFF, Centro Interdipartimentale di Ricerca in Farmacoeconomia e Farmacoutilizzazione, Università degli Studi di Napoli Federico II 


\section{Tabella I}

Costi unitari

*Media ponderata per la frequenza di ospedalizzazioni come risulta dalle schede di dimissione ospedaliera (SDO 2003)[8]

\begin{tabular}{lcc}
\hline \multicolumn{1}{c}{ Variabili } & DRG [7] & Costi unitari (euro) \\
\hline Infarto miocardico fatale e non fatale & $121,122,123$ & $4.175,13^{*}$ \\
Rivascolarizzazioni coronariche & $106,107,112$ & $7.996,28^{\star}$ \\
Angina instabile & 140 & $2.179,45$ \\
Ictus fatale e non fatale & 14 & $3.926,62$ \\
Pravastatina $40 \mathrm{mg}$ & - & 2,33 \\
Atorvastatina $80 \mathrm{mg}$ & - & 1,56 \\
\hline
\end{tabular}

L'end-point primario dello studio è costituito dal tempo di insorgenza di uno dei seguenti eventi: morte per qualsiasi causa, infarto del miocardio, angina instabile che richieda l'ospedalizzazione, rivascolarizzazione con bypass aortocoronarico o angioplastica [1].

Su tali basi abbiamo eseguito una valutazione economica utilizzando l'analisi di costo/efficacia [2-3] che ha raffrontato le conseguenze economiche e cliniche dell'utilizzo di una terapia intensiva a base di atorvastatina $80 \mathrm{mg} / \mathrm{die}$ rispetto alla terapia standard con pravastatina $40 \mathrm{mg} / \mathrm{die}$.

La prospettiva assunta è stata quella del Sistema Sanitario Nazionale (SSN) italiano, adottando il profilo temporale dello studio PROVE-IT che indica 24 mesi di follow-up medio. Tutte le analisi sono riferite a ipotetiche coorti di 1.000 pazienti $[4,5]$.

L'analisi di costo/efficacia è stata condotta calcolando l'ICER (rapporto costo/efficacia incrementale) come rapporto tra la differenza nei costi delle due alternative in relazione alla differenza di efficacia ed espresso come costo per morti evitate.

\section{Effetti}

Gli effetti sono stati identificati, misurati e quantificati mediante l'utilizzo delle evidenze dello studio PROVE-IT ed espressi come morti prevenute.

\section{Costi}

Coerentemente con la prospettiva d'analisi, sono stati identificati, misurati e quantificati i costi diretti sanitari.

Per il calcolo del costo della terapia farmacologica si è utilizzato lo schema terapeutico seguito nello studio PROVE-IT. Tale schema prevedeva che i pazienti che rispettavano i criteri di eleggibilità fossero randomizzati a ricevere o $40 \mathrm{mg} /$ die di pravastatina o $80 \mathrm{mg} / \mathrm{die}$ di atorvastatina. Alla fine dei 24 mesi il 33\% nel gruppo con pravastatina e il $30,4 \%$ nel gruppo con atorvastatina ha interrotto il trattamento in modo definitivo a causa di effetti collaterali, preferenze del paziente o altri motivi [1].

Il costo della terapia farmacologica è stato quantificato sulle basi della dose giornaliera e della durata del trattamento, utilizzando il prezzo di cessione all'SSN dei farmaci [6]. Per quantificare il costo di atorvastatina $80 \mathrm{mg}$, è stato ipotizzato un flat price sul prezzo di atorvastatina $40 \mathrm{mg}$.

I costi delle ospedalizzazioni sono stati calcolati in base al sistema tariffario DRG (Diagnosis Related Group) [7], come logica conseguenza della prospettiva adottata (la Tabella I riporta le tariffe associate a ogni evento clinico). È stato inoltre necessario tenere in considerazione il fatto che, per ogni evento clinico valutato, potevano esistere più tariffe, in relazione alla gravità dello stato di salute del paziente alla dimissione: ad esempio, nel caso della rivascolarizzazione, si è utilizzato il costo medio pesato per la frequenza di evento in accordo con le più recenti informazioni disponibili sui ricoveri ospedalieri in Italia, poiché dati sulla gravità non erano disponibili [8].

Inoltre nell'analisi non sono stati considerati i costi associati agli eventi avversi da farmaco, in quanto non erano disponibili informazioni dettagliate a riguardo.

I costi oltre i primi 12 mesi sono stati scontati con un tasso del $3 \%$; per semplicità sono stati riportati solo i risultati scontati. I costi sono espressi in euro 2006.

\section{Sensibilità}

La robustezza dei risultati ottenuti è stata valutata attraverso un'analisi di sensibilità sul prezzo dei farmaci $( \pm 10 \%)$, sui costi delle ospedalizzazioni e delle procedure $( \pm 10 \%)$.

Un'ulteriore analisi di sensibilità è stata condotta sulle procedure di sconto facendo variare il tasso di sconto annuale dal 5\% allo $0 \%$. È stata infine condotta un'analisi volta a identificare il prezzo soglia di atorvastatina che determinasse il medesimo costo per i due trattamenti in studio [9-11].

\section{RISULTATI}

L'obiettivo dello studio PROVE-IT consisteva nel valutare se le statine fossero efficaci nel ridurre gli eventi cardiaci e se l'abbassamento marcato del colesterolo fosse in grado di fornire benefici aggiuntivi in pazienti con sindrome coronaria acuta. Dai risultati emerge 


\begin{tabular}{|c|c|c|c|c|c|}
\hline \multirow{2}{*}{ Variabili } & \multicolumn{2}{|c|}{ Pravastatina* } & \multicolumn{2}{|c|}{ Atorvastatina* } & \multirow[b]{2}{*}{ Differenza* } \\
\hline & Numero & Costi totali & Numero & Costi totali & \\
\hline Infarto miocardico fatale e non fatale & 74 & $291.478,80$ & 66 & $259.967,58$ & $-31.511,22$ \\
\hline Rivascolarizzazioni coronariche & 188 & $1.418 .244,74$ & 163 & $1.229 .648,37$ & $-188.596,38$ \\
\hline Angina instabile & 51 & $104.862,97$ & 38 & $78.133,2$ & $-26.729,78$ \\
\hline Ictus fatale e non fatale & 10 & $37.044,52$ & 10 & $37.044,52$ & 0 \\
\hline Morti per eventi coronarici & 14 & & 11 & & \\
\hline Morti per tutte le cause & 32 & & 22 & & \\
\hline Costi totali per ospedalizzazioni & & $1.851 .631,03$ & & $1,604,793.66$ & $-246,837.37$ \\
\hline Costo terapia farmacologica & & $1.300 .814,63$ & & $870,404.80$ & $-430.409,83$ \\
\hline Costi totali & & $3.152 .445,66$ & & $2.475 .198,46$ & $-677.247,20$ \\
\hline
\end{tabular}

\section{Tabella II}

Analisi dei costi (in euro), valori scontati al 3\%

* Dati riferiti ad una ipotetica coorte di 1.000 soggetti

che i pazienti sottoposti a regime terapeutico con elevate dosi di atorvastatina hanno mostrato un rischio di morte per eventi coronarici maggiori ridotto rispetto ai pazienti in trattamento con dosi standard di pravastatina. Una terapia aggressiva mostra benefici anche per quanto riguarda gli eventi cardiovascolari, portando ad una riduzione del $29 \%$ nella frequenza di insorgenza di angina instabile e una riduzione del 14\% nella frequenza di ricorsi a procedure di rivascolarizzazione. La riduzione del tasso di mortalità $(28 \%)$ per qualsiasi causa era al limite della significatività suggerendo che una terapia ipocolesterolemizzante aggressiva è importante non solo per ridurre il rischio di ischemie ricorrenti, ma anche per ridurre il rischio di eventi fatali.

Il costo della terapia con pravastatina e atorvastatina nei 24 mesi è stato rispettivamente di circa 1,3 milioni di euro e di 870.404 euro per 1.000 pazienti. I costi totali delle ospedalizzazioni per eventi fatali e non è stato stimato pari a circa 1,9 milioni di euro nel gruppo trattato con pravastatina e pari a circa 1,6 milioni di euro nel gruppo trattano con atorvastatina (Tabella II).

Nel complesso il costo per 1.000 pazienti trattati per un periodo di 2 anni è stato stimato pari a 3.152.445 euro nel gruppo trattato con pravastatina e di 2.475.1981 euro nel gruppo trattato con atorvastatina, generando così un risparmio di 677.247 euro che rappresenta il $27 \%$ dei costi totali a carico del gruppo con pravastatina. Di conseguenza il trattamento con atorvastatina risulta dominante rispetto al trattamento con pravastatina, essendo contemporaneamente sia maggiormente efficace sia meno costoso.

I risultati dell'analisi di sensibilità mostrano come la valutazione rimanga sostanzialmente stabile al variare $\mathrm{di} \pm 10 \%$ i costi delle ospeda- lizzazioni e del trattamento. Il trattamento con atorvastatina rimane dominante anche nell'ipotesi più sfavorevole.

Infine, in accordo con l'analisi soglia, il prezzo di atorvastatina $80 \mathrm{mg}$ tale per cui i costi dei due trattamenti si eguagliano è 2,77 euro, ossia il $43,7 \%$ in più del prezzo utilizzato nell'analisi principale.

\section{DISCUSSIONE}

Il presente studio fornisce la prima valutazione economica dello studio PROVE-IT adattato alla realtà italiana. Esso ha permesso di dimostrare come il trattamento con elevate dosi di atorvastatina porti a una diminuzione dei costi diretti a carico del SSN che supera il costo addizionale dovuto al trattamento con atorvastatina: il risparmio globale è quindi di 677.247 euro per 1.000 pazienti trattati.

La maggior parte del risparmio è attribuibile alla riduzione del costo della terapia farmaco-

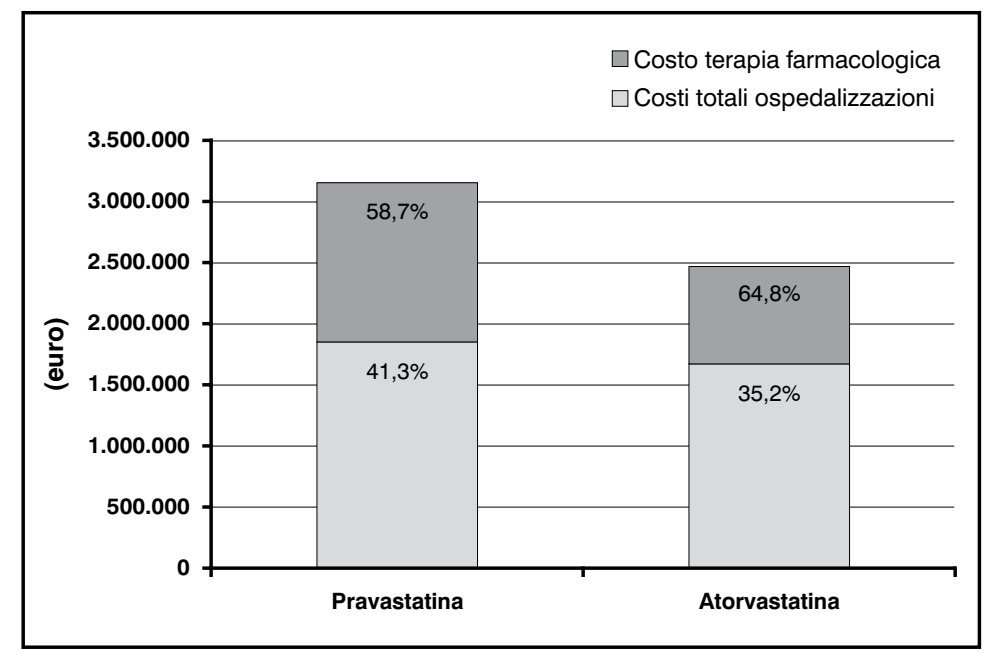

Figura 1

Costo per 1.000 soggetti per 2 anni 
logica (circa 2/3 del risparmio totale), seguita dalla riduzione nell'incidenza delle procedure di rivascolarizzazione. Inoltre l'analisi di sensibilità mostra che la terapia con atorvastatina rimane dominante anche nelle condizioni più sfavorevoli.

Lo studio presenta tuttavia alcuni limiti potenziali. Il primo è rappresentato dal fatto che non sono tenuti in considerazione $i$ costi legati alla gestione del paziente non in regime di ricovero quali ad esempio l'assistenza domiciliare e le terapie farmacologiche concomitanti. Il secondo è che non sono state incluse le conseguenze indirette di un evento cardiovascolare quali la perdita o il guadagno di produttività o conseguenze intangibili quali la compromissione della qualità di vita associata allo stato di salute. Il motivo è legato al fatto che queste informazioni non erano a nostra disposizione.

Sarebbe comunque interessante valutare come la terapia con statine possa influenzare la produttività dei pazienti. Si ipotizza che i pazienti in trattamento con pravastatina abbiano subito una maggiore riduzione nella capacità lavorativa a causa delle più frequenti ospedalizzazioni, quindi si può dire che l'introduzione nella valutazione economica anche dei costi indiretti avrebbe ulteriormente aumentato i benefici economici derivanti dall'utilizzo di dosi elevate di atorvastatina.

È anche interessante notare che il costo della terapia farmacologica è stato probabilmente sovrastimato. Infatti per mancanza di informazioni relative alla durata del ricovero non è stato possibile tenere in considerazione il fatto che il costo della terapia farmacologica è compreso nelle tariffe DRG per il periodo della durata del ricovero. Per cui una parte dei costi relativi alla terapia potrebbero essere stati quantificati due volte.

In accordo con i nostri risultati, il trattamento con dosi elevate di atorvastatina in soggetti con storia pregressa di infarto del miocardio porta in generale ad un risparmio per il SSN. La conoscenza degli effetti non solo clinici ma anche economici che derivano dall'utilizzo di queste strategie terapeutiche può essere di aiuto al fine di una allocazione più razionale delle risorse.

\section{BIBLIOGRAFIA}

1. Cannon CP, Braunwald E, McCabe CH, Rader DJ, Rouleau JL, Belder R et al. The pravastatin or atorvastatin evaluation and infection therapy-thrombolysis in myocardial infarction 22 investigators. Intensive versus moderate lipid lowering with statins after acute coronary syndromes. N Engl J Med 2004; 350: 1495-504

2. Weinstein MC, Stason WB. On the foundations of cost-effectiveness analysis for health and medical practices. N Engl J Med 1977; 296: 716-21

3. Drummond MF, Stoddart GL, Torrance GW. Methods for economic evaluation of health care programmes. Oxford: Oxford University Press, 1997

4. Mantovani LG, Belisari A, Dobrilla G. Valutazione economica del lansoprazolo nel trattamento dei pazienti affetti da malattia da reflusso esofageo. PharmacoEconomics Italian Research Articles 1999; 1: 43-51

5. Scalone L, Mantovani LG. Valutazione economica della terapia con lisinopril ad alto verso basso dosaggio nel trattamento dei soggetti con scompenso cardiaco cronico. Pharmacoeconomics Italian Research Articles 2002; 4: 45-55.

6. L'Informatore Farmaceutico. Milano: OEMF, 2006

7. Decreto ministeriale 14 dicembre 1994 in tema di "Tariffe delle prestazioni di assistenza ospedaliera". Supplemento ordinario alla Gazzetta Ufficiale n. 209, 8 settembre 1997

8. http://www.ministerosalute.it/programmazione/sdo/sdo.jsp

9. Briggs A, Sculpher M, Buxton M. Uncertainty in the economic evaluation of health care technologies: the role of sensitivity analysis. Health Econ 1994; 3: 95-104

10. Garattini L, Grilli R, Scopelliti D, Mantovani L. A proposal for Italian guidelines in pharmacoeconomics. Pharmacoeconomics 1995; 7: 1-6

11. Capri S, Ceci A, Terranova L, Merlo F, Mantovani LG. Guidelines for economic evaluation in Italy: recommendation from the Italian group of pharmacoeconomic studies. Drug Inf J 2001; 35: 189-201 